\title{
Variations in Apelin Expression Levels during Achilles Tendon Healing
}

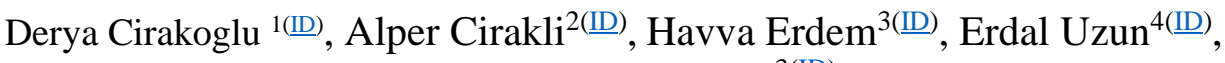 \\ Muruvvet Akcay Celik ${ }^{3(\underline{I D})}$ \\ ${ }^{1}$ Department of Phyisical Medicine and Rehabilitation, Ordu University Training Hospital, Ordu, Turkey \\ ${ }^{2}$ Department of Orthopedic and Traumatology, Ordu University Training Hospital, Ordu, Turkey \\ ${ }^{3}$ Department of Pathology, Ordu University Training Hospital, Ordu, Turkey \\ ${ }^{4}$ Department of Orthopedic and Traumatology, Kayseri Training Hospital, Kayseri, Turkey
}

Copyright@ Author(s) - Available online at https://dergipark.org.tr/en/pub/mbsjohs

Content of this journal is licensed under a Creative Commons Attribution-NonCommercial 4.0 International License,

Received: 15 February 2021, Accepted: 2 April 2021, Published online: 30 April 2021

(C) Ordu University Institute of Health Sciences, Turkey, 2021

\begin{abstract}
Objective: Research in recent years focused on the role of the apelin-APJ axis in pathologic fibrosis. The axis includes the parenchyma and mesenchymal cells of most organs. Reduction in parenchymal cells and activation of fibroblasts generally results in fibrosis of the organ. In this study, the aim was to reveal differences in apelin in tendons with primary repair.

Methods: The study used 15 male Wistar Albino rats. The tendons of the right and left legs of the rats were used. Rats were divided into 3 groups containing 5 rats each. Group 1 was the sham group; Group 2 had tendon repair performed and were sacrificed 3 weeks later; and Group 3 had tendon repair and were sacrificed 6 weeks later. Under anesthesia, bilateral tendonectomy was performed and surgically repaired. Tendons were removed in the 3rd and 6th weeks. Samples were immunohistochemically stained for apelin and stain degree was assessed from 0 to 4 with a light microscope. The scores were compared with the Friedman test. Results: The results of statistical evaluation identified that the sham group had statistically significantly higher levels compared to the scores in the other two groups. There was no significant difference identified between the groups sacrificed in the 3rd and 6th weeks.

Conclusion: This study concluded that apelin may be beneficial for tendon healing and that apelin levels may increase with fibrosis.
\end{abstract}

Key words: Apelin, repair, fibrosis

Suggested Citation Cirakoglu D, Cirakli A, Erdem H, -Uzun E, Akcay Celik M. Variations in apelin expression levels during achilles tendon healing. Mid Blac Sea J Health Sci, 2021;7(1):57-63

\section{Address for correspondence/reprints:}

Derya Çirakoglu

Telephone number: +90 5053156795

E-mail: drderya79@gmail.com

\section{Introduction}

In the USA nearly 300 thousand tendon and ligament repair operations are performed each year (1). In spite of surgical intervention, the healing process of tendons is slow as tendons and ligaments are hypocellular and hypovascular (2). Even 1 year later, the structure and function of injured tendons is lower than uninjured tendons. The amelioration response is divided into 3 intersecting stages of inflammation, proliferation/repair and remodeling (3). After surgical repair of the tendon, generally a 
short inflammatory phase lasting up to a week begins. This is followed by a proliferative phase lasting several weeks. Then, the remodeling phase lasting several months occurs (4). During the inflammatory phase, vascular permeability increases and the entry of inflammatory cells into the healing area occurs. These cells produce a range of growth factors and cytokines involved in duties and proliferation of fibroblasts and macrophages. During the proliferation and remodeling phases of amelioration, fibroblasts multiply and begin production, storage and crosslinking of collagen (4).

Evidence in recent times proposed that inflammation modulation in the early stages following tendon repair may cause healing (5). Regulated inflammation is generally beneficial for tissue repair; however, excessive and permanent inflammation is known to cause damage. In fact, inflammatory cytokines attract fibroblasts to the repair area, with excessive inflammation causing poor clinical outcomes $(6,7)$.

Apelin is a peptide hormone. It is an endogenous ligand for the G-protein linked receptor called APJ. Apelin and APJ are commonly expressed in the heart, lungs, hypothalamus, adipose tissue, kidneys, muscles and other organs in humans (8). Apelin is initially synthesized as 77 amino acid precursors. Later it converts to several mature forms like apelin 13, apelin 17 and apelin 36 (9). Apelin 13 is the main form circulating in plasma and is considered to be mainly effective on physiology (9). Studies in recent years focused on the role of the apelin-APJ axis in pathologic fibrosis. Many organs contain parenchymal and mesenchymal cells. The reduction in parenchymal cells and activation of fibroblasts generally results in the development of fibrosis in the organ. The leading characteristics of organ fibrosis are production of inflammatory factors, secretion in the extracellular matrix and activation and proliferation of fibroblasts (10). Organ fibrosis forms in the kidneys, heart, lungs and liver. Fibrosis reduces the functions of the organ and causes organ failure. Recent studies show that the apelin-APJ axis is associated with renal fibrosis, myocardial fibrosis, liver fibrosis and pulmonary fibrosis. Additionally, the function of apelin-APJ in development of fibrosis is controversial. Many factors like transforming growth factor (TGF-B1) beta, angiotensin 2 (Ang2) and extracellular matrix (ECM) participate in the organ fibrosis process. These factors support cell proliferation and differentiation, wound healing and extracellular matrix production contributing to the development of fibrosis $(11,12)$. Some articles proposed that apelin-APJ inhibits renal and myocardial fibrosis via the TGF- $\beta$ route. Contrarily, the apelin-APJ axis increases liver fibrosis. There is no information about the presence or efficacy of apelin during the tendon healing process. This study aimed to research whether apelin contributes to healing after primary repair of tendons.

\section{Methods}

\section{Animals}

The minimum number of animals required in order to obtain statistically significant results were used in our study. Our study used fifteen Wistar albino rats weighing 250-300 g initially and aged from 4-8 weeks. During the experiment, rats were kept in 10/14-hour light/darkness cycles, with 3 subjects per cage at normal room temperature and humidity fed with standard pellet feed (Nükleon, Ankara, Turkey) and tap water. Temperature was measured with a thermometer and humidity with a hygrometer. Ventilation was provided by a room aspirator.

\section{Formation of the groups and experimental design}

Before beginning the experiment, animals were weighed and divided into 3 equal groups with similar animal weights in the group distributions (groups are explained below). A total of 15 male rats were used.

Sham group (5 rats): This group was sacrificed on the same day. The regions of the right and left Achilles tendons had skin and subdermal tissue entered with a $3 \mathrm{~cm}$ incision and full layer incision was made $0.5 \mathrm{~cm}$ proximal of the Achilles tendon adhesion point. The Achilles tendons were removed on the same day.

Group 1 (sf-3) (5 rats): This group was sacrificed after 21 days. The regions of the right and left Achilles tendons had skin and subdermal tissue entered with a $3 \mathrm{~cm}$ incision and full layer incision was made $0.5 \mathrm{~cm}$ proximal of the Achilles tendon adhesion point. Incisions were primarily repaired with the modified Kessler method using 4.0 polypropylene sutures (Propilen, Doğsan, Trabzon, Turkey). Later subdermal layers and skin were closed in retrograde manner. The Achilles tendons were removed 21 days later.

Group 2 (sf-6) (5 rats): This group was sacrificed after 42 days. The regions of the right and left Achilles tendons had skin and subdermal tissue entered with a $3 \mathrm{~cm}$ incision and full layer incision 
was made $0.5 \mathrm{~cm}$ proximal of the Achilles tendon adhesion point. Incisions were primarily repaired with the modified Kessler method using 4.0 polypropylene sutures (Propilen, Doğsan, Trabzon, Turkey). Later subdermal layers and skin were closed in retrograde manner. The Achilles tendons were removed 42 days later.

All procedures in the study were completed in accordance with ethical guidelines. Before surgery, rats were anesthetized with $3 \mathrm{mg} / \mathrm{kg}$ xylazine hydrochloride (Rompun(C), Bayer, Turkey) and 90 $\mathrm{mg} / \mathrm{kg}$ ketamine hydrochloride (Ketalar ${ }^{\circ}$, Eczacibas1, Turkey) administered intraperitoneally. After appropriate anesthesia was achieved, animals were shaved with a razor taking care not to harm the skin and skin was cleaned with polyvinyl pyrrolidone-iodine (Batticon $\mathbb{C}$, Adeka, Samsun, Turkey). The animal was covered with sterile compresses leaving the surgical field open. After the surgical procedure all animals had regular daily wound dressing performed.

Samples were taken from the tissues and then sections with a thickness of $5 \mu \mathrm{m}$ were prepared on poly-laminated slides. Slides were prepared for immunohistochemical study. A Leica Bond-Max IHK stain device (Vision Biosystems, Melbourne, Australia) was used for immunohistochemical study.

Sections were left at 60 degrees for 30 minutes. Then they were left at 72 degrees in Bond Devax solutions for deparaffinization. After washing with alcohol 3 times, they were washed 3 times with Bond Wash washing solution. They were left for 10 minutes at 100 degrees in the pre-processing solution previously defined for the antibody. Then, they were washed 3 times more in Bond Wash washing solution. Ten minutes of peroxide blockage was performed. Again, they were washed 3 times with Bond Wash washing solution. They were incubated with apelin (genetex GTX37465 (Polyclonal (1:300)). Then, they were washed 3 times with Bond Wash washing solution. They were treated with post-primer for 7 minutes. Then, after washing 3 times with Bond Wash washing solution, they were treated with the polymer for 7 minutes. Then, they were washed 2 times with Bond Wash washing solution and then with distilled water. They were incubated for 7 minutes with DAB and washed with distilled water 3 times. At the end of these processes, they were assessed with the degree procedure of Berta et al. (15).

The proportion of cells with cytoplasmic positivity was taken into account. Accordingly, 0 staining, $1+$ staining, 2 + staining, or 3 + staining categories were defined on the basis of no staining, $1 \%$ to $10 \%$ staining, $11 \%$ to $50 \%$ staining, and greater than $50 \%$ staining, respectively (Figure 1-3).

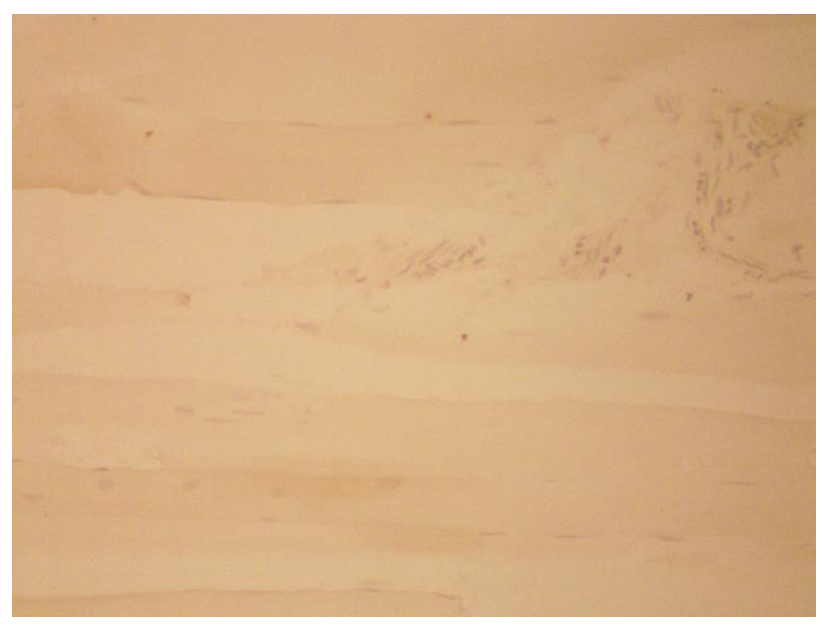

Figure 1. Sham group staining example (x400 magnification)

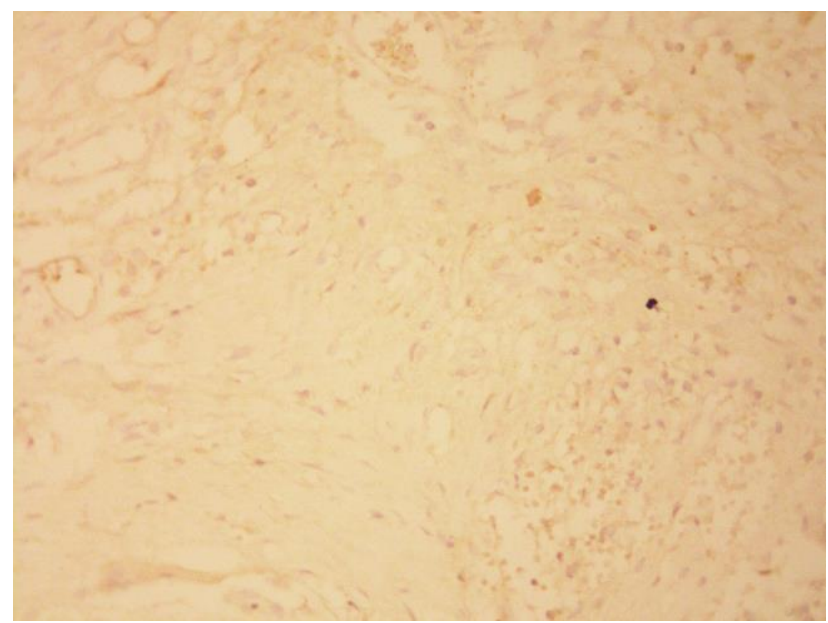

Figure 2. Sf-3 group staining example (x400 magnification)

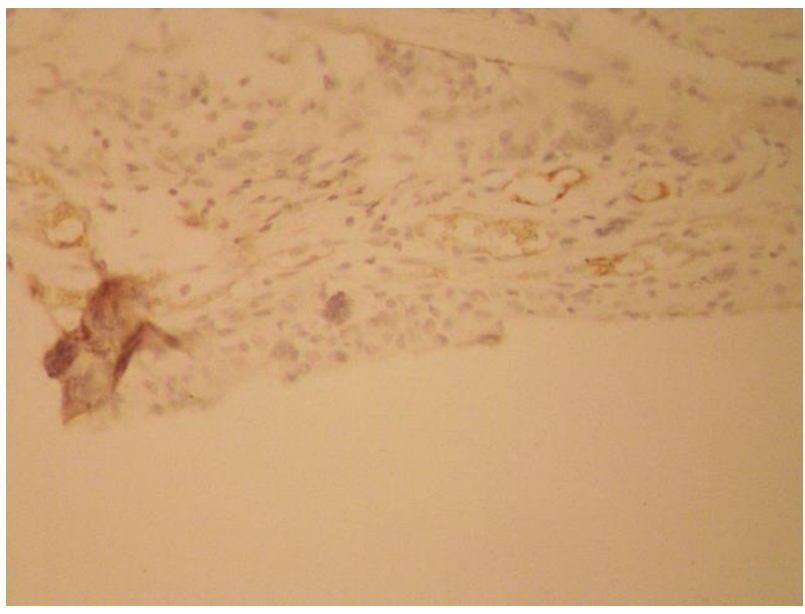

Figure 3. Sf-6 group staining example (x400 magnification) 
Statistical analysis

The SPSS v20 (IBM Inc., Chicago, IL, USA) computer program was used for statistical analysis. As each group included 5 subjects, comparisons were performed with the non-parametric Friedman test and the chi-square test for stain differences between the three groups and temporal changes in the sf- 3 and sf6 groups. Statistical significance was taken as $p \leq$ 0.05 .

\section{Ethics of the Study}

Our study was approved by the Experimental Animals Ethical Committee of the Faculty of Medicine (Date: 30.01.2018, Issue:03).

\section{Results}

The Friedman test results for comparison of the three groups found statistically significant differences between the degree of staining in the three groups with $\times 2=16.75$ and $p<0.001$. The group with most staining was Group sf-6 (mean rank: 2.70). Staining examples for the sham, sf- 3 and sf- 6 groups are seen in Figures 1, 2 and 3 (x400 magnification) (Graphic $1)$.

Additionally, as time passed between the sf- 3 and sf-6 groups, the chi-square test results for staining did not have a statistically significant difference with $\times 2=$ 1.01 and $\mathrm{p}=0.31$.

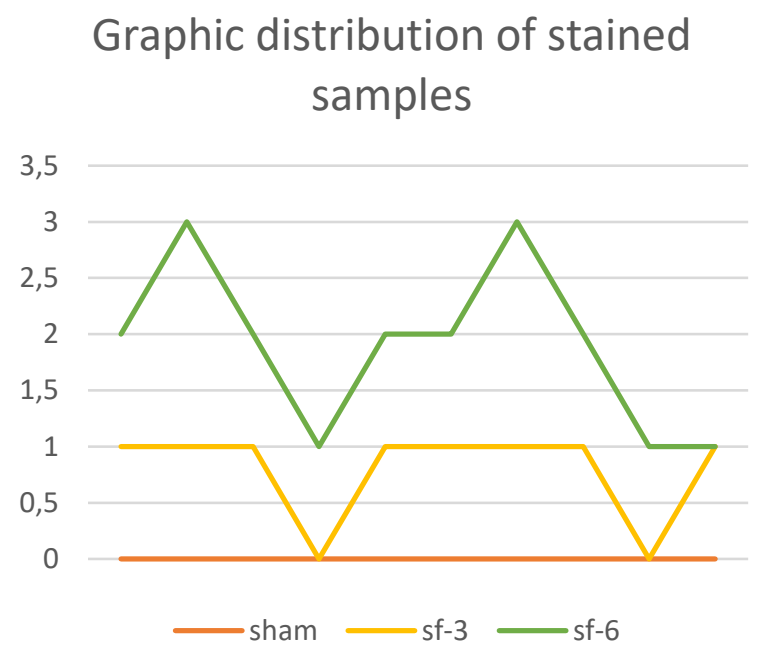

Graphic 1. Distribution of stained samples

\section{Discussion}

In our study, the apelin level was identified to increase with Achilles' tendon injury in rats. The Achilles tendon is the strongest and most used tendon in the body (13). Many bioactive molecules play a role in regulating the cellular response during tendon repair (14). A variety of growth factors are active in many stages of the healing process and are clearly upregulated after tendon injury, including insulin-like growth factor-I (IGF-I), TGF- $\beta$, bFGF, plateletderived growth factor (PDGF), vascular endothelial growth factor (VEGF), BMP, and connective tissue growth factor (CTGF) (15-17).

Tenoblasts and tenocytes are tendon-specific cell types forming the majority of cellular content in tendons (18). Tenocytes are accepted as being fibroblast-like cells producing necessary components like type I collagen and other ECM molecules during growth and healing of collagen fibers.

The resistance of the tendon is linked to the ability of the collagen molecules to organize and form a cross-linked structure (19). Type I collagen is dominant in ECM in tendons, while type III collagen is the second-most common and critical collagen type in pathologic tendons and tendon healing processes (20). The organization of type I collagen is accepted as being very important for the mechanical characteristics of tendons. Finally, research aimed to understand the role of growth factors, transcription factors and type I collagen-fibril distribution in regulation-linked mechanical forces. For example, TGF $\beta$ and FGF were shown to regulate collagenarchitectural formation within tendons during development $(21,22)$. Additionally, the transcription factors scleraxis, Mohawk homeobox protein, and zinc-finger protein early growth response protein 1 (EGR1) regulate type I collagen formation within tendons via modulation of COL1A1 and COL1A2 gene expression $(23,24)$. Extrinsic cells including neutrophils and macrophages playing a key role in cleaning debris release second generation cytokines passing into the next stage of the healing process (25).

The proliferative phase characterized by scattered accumulation of granulation tissue and a peak in type III collagen and DNA concentrations continues with the transition from type III to type I collagen in later stages (6).

The majority of organs contain parenchymal and mesenchymal cells. Reductions in parenchymal cells and activation of fibroblasts generally results in the development of organ fibrosis. Structural injury from continuing fibrosis reduces organ functions and finally causes organ failure. Many factors like TGF beta, angiotensin II (Ang-II) and extracellular matrix (ECM) participate in the organ fibrosis process (10,26-28). Research studies show the apelin-APJ axis is associated with renal, myocardial, liver and pulmonary fibrosis (26). However, this association may not be the same in all organs. Some articles proposed that apelin-APJ inhibits renal and myocardial fibrosis via the TGF beta pathway $(10,26-$ 
28). In vitro apelin-induced TGF beta in human proximal tubular epithelium cells inhibits the epithelial-mesenchymal transition. In the unilateral ureteral obstruction model, apelin treatment significantly reduced TGF beta 1 and its receptor simultaneous to expression of interstitial matrix components. Apelin may improve renal interstitial fibrosis by suppressing the tubular epithelialmesenchymal transition with the Smad proteindependent mechanism pathway (27). Canakci et al. reported apelin has a protective role in experimental renal IR injury (28). These findings lead to the consideration that apelin-APJ has potential renoprotective effects and may be an effective agent in delaying progression of CRF. Additionally, recent studies lead to consideration that the apelin/APJ system may be used for treatment of insulin resistance and type 2 diabetes (29).

Similarly, the apelin-APJ axis is thought to reduce myocardial fibrosis. After MI in mice, apelin 13 treatment ensured a reduction in the degree of cardiac hypertrophy and cardiac fibrosis compared to the sham group $(30,31)$. This effect of apelin was due to stimulation of bone marrow cells (BMC). Apelin stimulated bone marrow cells (BMC) and excessive expression of apelin by BMC increases the speed of cardiac repair and lessens cardiac fibrosis in post-MI mice (32). Strohbach et al. showed that plasma apelin-17 levels significantly fell in patients with acute myocardial infarctus (AMI) compared to the control group. They reported the platelet apelinergic system may be a new target for diagnostic and treatment purposes (33). While the apelin/APJ system has positive inotropic effect on the heart, it was also shown to ensure water excretion. Using this, it was proposed as a treatment choice for congestive heart failure accompanied by hyponatremia (34). Additionally, apelin was identified in breastmilk. It is thought to play a role in the foundation of the infant immune system. At the same time, activation of the apelin/APJ system was reported to improve muscle weakness linked to aging (35).

Angiogensin II (AII) and endothelin-1 (ET-1) increase the apelin expression in hepatic stellate cells (HSC). Increased apelin increases synthesis of collagen I and platelet-derived growth factor receptor $\beta$ (PDGFR $\beta$ ) closely associated with liver fibrosis (36). This data shows that apelin-APJ may be an important mediator of liver fibrogenesis. Additionally, apelin and APJ levels clearly increase in cirrhotic liver (37). Apelin causes collagen I stimulation leading to fibrosis in LC (36). However, there are studies proposing the opposite to this. Studies by Owen et al. (38) reported that plasma apelin levels significantly reduced in both the early stage of liver fibrosis and the late stage of liver cirrhosis.

Our study is the first to reveal increased apelin levels in Achilles tendon injury. This study observed the apelin expression in the 3-and 6-week groups was greater compared to the sham group. This difference in apelin may be important in the healing process after primary repair, angiogenesis, anti-inflammatory cytokines and in the remodeling process. In the literature, it was observed that apelin ensures collagen I increase. For tendon repair, provision of collagen I instead of collagen III is necessary for healthy repair to occur. In our study, increased levels of apelin in the Achilles tendon after tendon repair may target healthy repair.

\section{Conclusions}

This study observed the apelin expression in the 3and 6-week groups was higher compared to a control group. In the healing process after primary repair, this difference in apelin may be important for angiogenesis, anti-inflammatory cytokines and in the remodeling process. This difference in apelin during tendon healing, especially, appears to be a candidate as a valuable topic for further research.

Ethics Committee Approval: This study was approved by the Experimental Animals Ethical Committee of the Faculty of Medicine (Date: 30.01.2018, Issue:03).

Peer-review: Externally peer-reviewed.

\section{Author Contributions:}

Concept: D.C, A.C, HE, Design: D.C, A.Ç, H.E, E.U; Literature search: MAC, Data Collection and Processing: D.C, A.Ç, H.E, E.U MAC; Analysis or Interpretation: D.C, A.Ç, H.E, E.U MAC; Writing: D.C, H.E.

Conflict of Interest: No conflict of interest was declared by the authors.

Financial Disclosure: The authors declared that this study hasn't received no financial support.

\section{References}

1. Pennisi E. Tending tender tendons. Science 2002; 295 (5557): 1011.

2. Liu CF, Aschbacher-Smith L, Barthelery NJ, Dyment N, Butler D, Wylie C. What We Should Know Before Using Tissue Engineering Techniques to Repair Injured Tendons: A Developmental Biology Perspective. Tissue Eng Part B-Rev 2011; 17(3): 165-76. 
3. Hope M, Saxby TS. Tendon healing. Foot and ankle clinics. 2007; 12(4): 553-67.

4. Voleti PB, Buckley MR, Soslowsky LJ. Tendon healing: repair and regeneration. Annu Rev Biomed Eng 2012; 14: 47-71.

5. Hays PL, Kawamura S, Deng XH, Dagher E, Mithoefer K, Ying $L$ et al. The role of macrophages in early healing of a tendon graft in a bone tunnel. J Bone Joint Surg Am 2008; 90(3): 565-79.

6. Sugg KB, Lubardic J, Gumucio JP, Mendias CL. Changes in macrophage phenotype and induction of epithelial-to-mesenchymal transition genes following acute Achilles tenotomy and repair. J Orthop Res 2014; 32: 944-51.

7. Lichtnekert J, Kawakami T, Parks WC, Duffield JS. Changes in macrophage phenotype as the immune response evolves. Curr Opin Pharmacol 2013; 13(4): 555-64.

8. Tatemoto K, Hosoya M, Habata Y, Fujii R, Kakegawa T, Zou MX, et al. Isolation and characterization of a novel endogenous peptide ligand for the human APJ receptor, Biochem. Biophys. Res. Commun 1998; 251(2): 471-76.

9. Pitkin SL, Maguire JJ, Bonner TI, Davenport AP. Apelin receptor nomenclature, distribution, pharmacology, and function. Pharmacol Rev 2010; 62(3): 331-42.

10. Ghosh AK, Quaggin SE, Vaughan DE. Molecular basis of organ fibrosis: potential therapeutic approaches. Exp Biol Med 2013; 238: 461-81.

11. Brand T, Schneider MD. The TGF beta superfamily in myocardium: ligands, receptors, transduction and function. J Mol Cell Cardiol 1995; 27: 5-18.

12. Brand T, Schneider MD. Transforming growth factor-beta signal transduction. Circ Res 1996; 78: 173-79.

13. Calleja M, Connell DA. The achilles tendon. Seminars in Musculoskeletal Radiology 2010; 14(3): 307-22.

14. Andia I, Sanchez M, Maffulli N. Tendon healing and platelet-rich plasma therapies. Expert Opinion on Biological Therapy 2010; 10(10): 1415-26.

15. Chen B, Wang B, Zhang WJ, Zhou G, Cao Y, Liu W. In vivo tendon engineering with skeletal muscle derived cells in a mouse model. Biomaterials 2012; 33(26): 6086-97.

16. Chen CH, Cao Y, Wu YF, Bais AJ, Gao JS, Tang JB. Tendon healing in vivo: Gene expression and production of multiple growth factors in early tendon healing period. The Journal of Hand Surgery 2008; 33(10): 1834-42.
17. Wurgler-Hauri CC, Dourte LM, Baradet TC, Williams GR, Soslowsky LJ. Temporal expression of 8 growth factors in tendon-to-bone healing in a rat supraspinatus model. J Shoulder Elbow Surg 2007; 16(5): 198-203.

18. Docheva D, Müller SA, Majewski M, Evans CH. Biologics for tendon repair. Adv Drug Deliv Rev. 2015; 84: 222-39.

19. Zhang G, Young BB, Ezura Y, Favata M, Soslowsky LJ, Chakravarti S, et al. Development of tendon structure and function: regulation of collagen fibrillogenesis. J Musculoskelet Neuronal Interact 2005; 5(1): 5-21.

20. Riley G. The pathogenesis of tendinopathy: a molecular perspective. Rheumatology (Oxford) 2004; 43(2): 131-42.

21. Lorda-Diez CI, Montero JA, Martinez-Cue C, Garcia-Porrero JA, Hurle JM. Transforming growth factors $\beta$ coordinate cartilage and tendon differentiation in the developing limb mesenchyme. J Biol Chem 2009; 284(43): 2998896.

22. Pryce BA, Watson SS, Murchison ND, Staverosky JA, Dunker N, Schweitzer R. Recruitment and maintenance of tendon progenitors by TGF $\beta$ signaling are essential for tendon formation. Development 2009; 136(8): 1351-61

23. Murchison ND, Price BA, Conner DA, Keene DR, Olson EN, Tabin CJ, et al. Regulation of tendon differentiation by scleraxis distinguishes force-transmitting tendons from muscleanchoring tendons. Development 2007; 134(14): 2697-708.

24. Guerquin MJ, Charvet B, Nourissat G, Havis E, Ronsin O, Bonnin MA et al. Transcription factor EGR1 directs tendon differentiation and promotes tendon repair. J Clin Invest 2013; 123(8): 356476.

25. Liu W, Watson SS, Lan Y, Keene DR, Ovitt CE, Liu $\mathrm{H}$ et al. The atypical homeodomain transcription factor Mohawk controls tendon morphogenesis. Mol Cell Biol 2010; 30(20): 4797-807.

26. Huang S, Chen L, Lu L, Li L. The apelin-APJ axis: A novel potential therapeutic target for organ fibrosis. Clin Chim Acta. 2016; 456(1): 81-88.

27. Wang LY, Diao ZL, Zhang DL, Zheng JF, Zhang QD, Ding JX et al. The regulatory peptide apelin: a novel inhibitor of renal interstitial fibrosis. Amino Acids 2014; 46: 2693-704. 
28. Canakci E, Karataş A, Erdem H, Celik M.A, Cirakoglu A, Cebeci Z. Effect of ischemic changes in renal tissues on apelin level. International Journal of Scientific Research April 2018; 7:5:82-85

29. Antushevich H, Wójcik M. Review: Apelin in disease. Clin Chim Acta. 2018 Aug; 483:241-48

30. Siddiquee K, Hampton J, Khan S, Zadory D, Gleaves L, Vaughan DE, et al. Apelin protects against angiotensin II induced cardiovascular fibrosis and decreases plasminogen activator inhibitor type-1 production. J Hypertens 2011; 29: 724-31.

31. Li L, Zeng H, Chen JX. Apelin-13 increases myocardial progenitor cells and improves repair postmyocardial infarction. Am J Physiol Heart Circ Physiol. 2012; 303: 605-18.

32. Li L, Zeng H, Hou X, He X, Chen JX. Myocardial injection of apelin-overexpressing bone marrow cells improves cardiac repair via upregulation of Sirt3 after myocardial infarction. PLoS One 2013; 8(9) e71041.

33. Strohbach A, Böhm A, Mahajan-Thakur S, Wirtz $\mathrm{C}$, Wetzel $\mathrm{H}$, Busch MC et al. Platelet apelin receptor expression is reduced in patients with acute myocardial infarction. Vascul Pharmacol 2021 Feb;136: 106808

34. Hu G, Wang Z, Zhang R, Sun W, Chen X. The Role of Apelin/Apelin Receptor in Energy Metabolism and Water Homeostasis: A Comprehensive Narrative Review Front Physiol. 2021 Feb 10;12: 632886.

35. Kinjo $T$, Higashi $H$, Uno $K$, Kuramoto $N$. Apelin/Apelin Receptor System: Molecular Characteristics, Physiological Roles, and Prospects as a Target for Disease Prevention and Pharmacotherapy. Curr $\quad$ Mol Pharmacol.2021;14(2):210-219.

36. Melgar-Lesmes P, Casals G, Pauta M, Ros J, Reichenbach V, Bataller R et al. Apelin mediates the induction of profibrogenic genes in human hepatic stellate cells. Endocrinology 2010; 151: 5306-14.

37. Yokomori H, Oda M, Yoshimura K, Machida S, Kaneko F, Hibi T et al. Overexpression of apelin receptor (APJ/ AGTRL1) on hepatic stellate cells and sinusoidal angiogenesis in human cirrhotic liver. J Gastroenterol 2011; 46: 222-31.

38. Owen NE, Nyimanu D, Kuc RE, Upton PD, Morrell NV, Alexander GJ et al. Plasma levels of apelin are reduced in patients with liver fibrosis and cirrhosis but are not correlated with circulating levels of bone morphogenetic protein 9 and 10. Peptides. 2021 Feb;136: 170440. 